\title{
PERFORMANCE OF THE AGS TRANSITION JUMP SYSTEM*
}

\author{
L.A. Ahrens, J.M. Brennan, J.W. Glenn, T. Roser, W.K. van Asselt" BNL, Upton, NY
}

\begin{abstract}
The transition jump system has been indispensable to the high intensity proton operation of the AGS complex. Nevertheless, transition crossing remains one of the major hurdles as the accelerator complex intensity is pushed upward. To enhance the performance of the system 'quadrupole pumping' in the Booster is used to minimize the necessary longitudinal dilution of the beam on the AGS injection porch. During the transition jump sextupole correctors at strategic locations are pulsed to minimize the effects of the chromatic non-linearity of the jump system. The available instrumentation for diagnosing the performance of the system will be described, along with performance at the recent record beam intensities.
\end{abstract}

\section{INTRODUCTION}

The gamma jump system at the AGS is in operation since 1994. It consists of three quadrupole doublets positioned symmetrical around the ring. When these quadrupoles are powered, they producc lattice perturbations resulting in a change of the transition energy. During acceleration of the beam in the AGS these quadrupoles are slowly ramped as the beam nears the transition energy of the unperturbed machine. The beam remains below transition in this way for a longer period. When the beam energy is well above the transition energy of the unperturbed machine, the currents in the quadrupoles are crowbarred to produce a fast change of the transition energy of the lattice and therefore an effective fast crossing of transition. Since the time constant of this crowbar is proportional to the inductance of the load each quadrupole magnet has its own charging supply and crowbar circuit. A more detailed description of the system can be found in $[1,2]$ and references cited there.

The large distortion to the machine lattice inherent to the jump implies that the beam momentum spread, hence longitudinal emittance, is the critical beam parameter near transition.

The following will outline the strategies, which have been employed during the high intensity proton running period for the High Energy Physics program of FY98 and FY99. The first involves RF gymnastics in the Booster aimed at reducing the longitudinal emittance of the beam in the AGS. The second involves powering sextupoles using newly installed hardware to counter the non-linear effects of the transition jump system.

\section{REDUCTION OF THE BEAM DILUTION IN THE AGS}

Optimizing beam intensity in the AGS involves a compromise between conflicting needs to create the most stable conditions on the long injection porch, and to produce a beam with minimal momentum spread for the transition jump. Beam properties relevant to front porch stability issues include the peak beam current, the momentum spread as well as tranverse dilution. The beam has been longitudinally mismatched upon transfer from the Booster to the AGS in previous years and an RF cavity with a frequency about forty time that of the accelerating RF has been used, which smooths and speeds filamentation of the beam increasing the longitudinal emittance and the beam stability $[3,4]$. At transition this results in increased beam losses, because the beam size increases so much, which is a

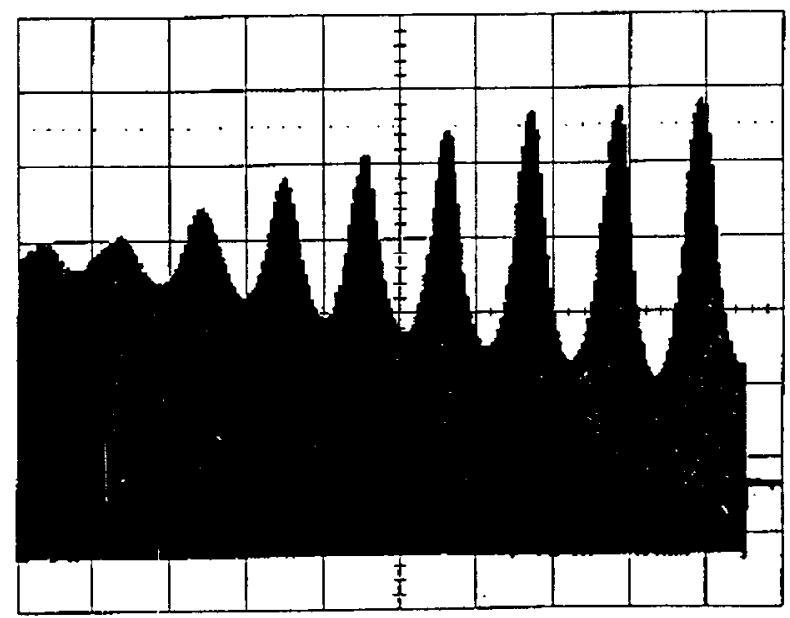

Figure 1: The wall current monitor signal showing the peak current density variations in the Booster before transfer to the AGS. Horizontal scale is $1 \mathrm{~ms} / \mathrm{s}$

* Work performed under the auspices of the U.S. Department of Energy

\# Email: vanasselt@bnl.gov 
Result of the large modulation of the dispersion function during the pulsing of the transition jump quadrupoles [1.2]. For this running period the peak bunch intensity and momentum spread of the bunches transferred from the Booster to the AGS has been reduced by so-called quadrupole pumping. a process where the RF voltage is modulated before the beam transfers to the AGS. In this way the lower peak densities can be obtained in the AGS without the emittance increase associated with the dilution of the high frequency cavity. Figure 1 shows the wall current monitor signal in the Booster during the last $10 \mathrm{~ms}$ before transfer to the AGS. It is seen that the peak density is reduced to approximately $60 \%$ from the value before the quadrupole pumping process started.

However, operation of the highest intensity beams have been obtained with a significant longitudinal mismatch intentionally present between the Booster and the AGS, implying that some momentum spread increase was needed on the injection porch.

\section{NON-LINEAR EFFECTS OF THE TRANSITION JUMP SYSTEM}

The second order $\gamma_{t}$-jump scheme used in the AGS significantly distorts the machine lattice. In [5] Wei et al. described measurements and simulations of the large chromatic non-linearity. which accompanies the powering of the transition jump quadrupoles. Wei proposed the use of a current of about $100 \mathrm{~A}$ in the twelve superperiod symmetric horizontal chromaticity sextupoles in the AGS to minimize the nonlinear effects of the jump system. Subsequent measurements, using a longitudinally very small beam. confirmed this prediction [6].

However, attempts to implement this plan using the high intensity beam over the past several years have consistently resulted in increased beam losses at transition even for sextupole currents well below $100 \mathrm{~A}$.

Although this is not rigorously understood, it is known that the beam equilibrium orbit is significantly offset from the sextupole centers. The resulting distortions may be responsible for the increased losses, which motivated attempting to reduce the number of sextupoles involved in the correction. Closer investigations of the effect of the sextupoles with the computer simulation code MAD showed that by choosing only three of the twelve sextupoles, those located near maxima of the distorted dispersion function inherent to the jump (B13, F13 and J13), an equally effective reduction in the nonlinearity could be achieved. Figure 2 shows some results of these simulations. The transition energy is plotted as a function of the momentum error of the beam in the AGS for various currents in these three sextupoles. These simulations also revealed some ill effects of the sextupole correction. Figure 3 shows the

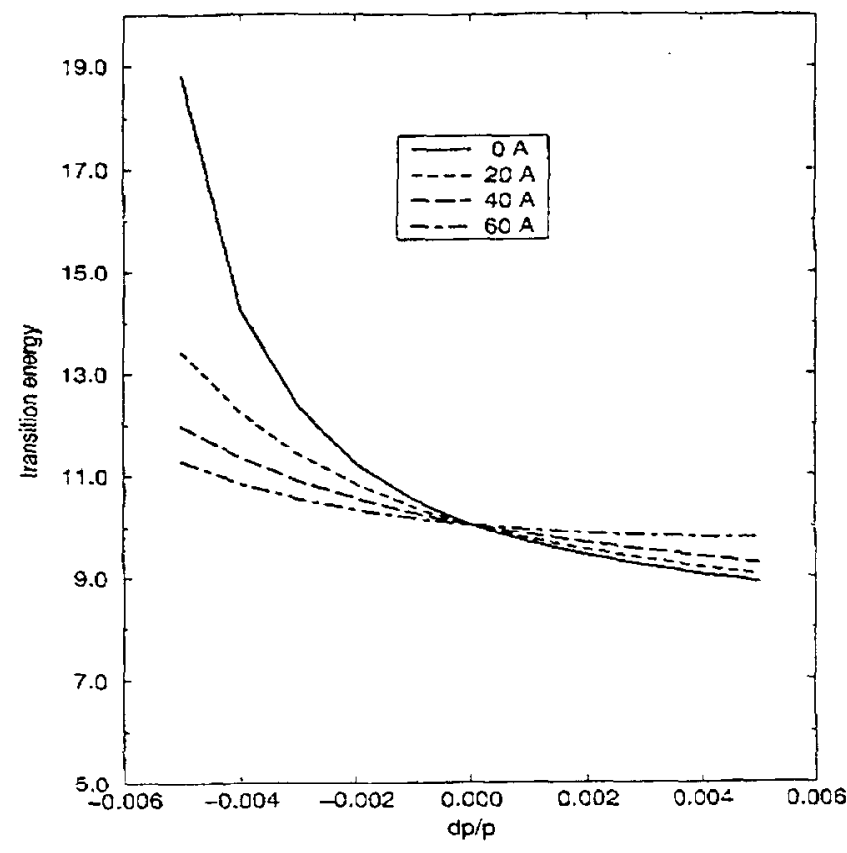

Figure 2: The transition energy versus mumentum with the sextupole current as parameter

maxima in the horizontal beta function as a function of the momentum crror for various values of the currents in the horizontal sextupoles.

To introduce sextupole correction near transition with minimal effect on the orbit and independent of the regular chromaticity correction, additional (floating) power supplies

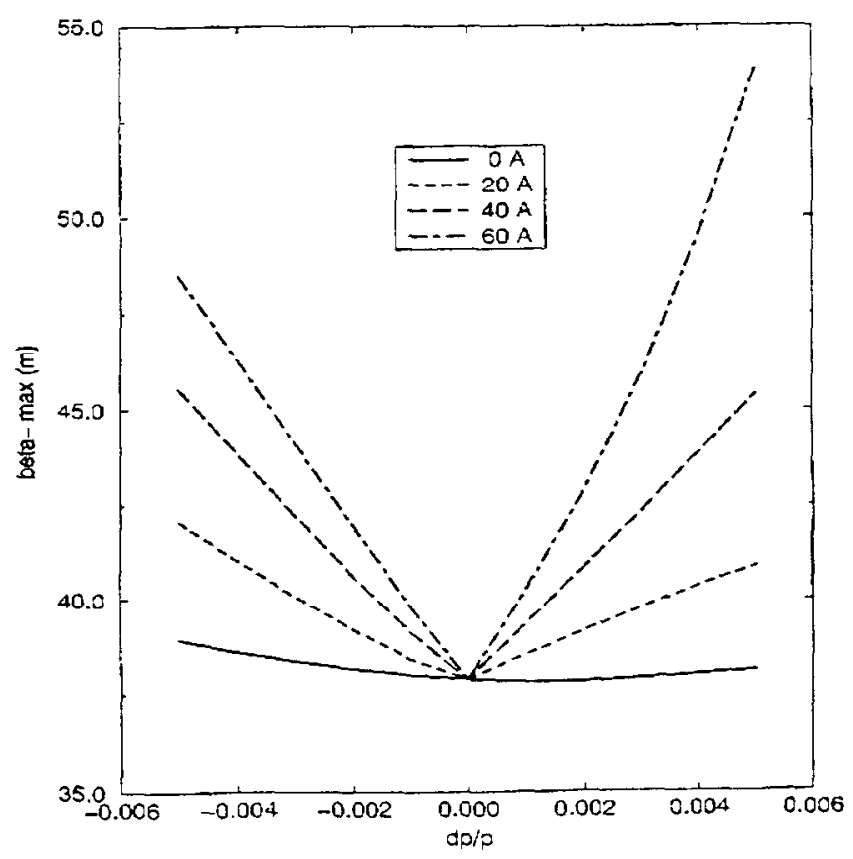

Figure 3: The maximum horizontal beta function versus momentum for different sextupole settings 
have been installed for each of the three sextupoles at B I3, F13 and J13 in the AGS ring. In pulsed mode the supplies are able to output $100 \mathrm{~A}$.

\section{DISCUSSION}

During the recent high intensity proton run the AGS complex has reached record intensities above $70^{*} 10^{12}$ proton per cycle (70 TP). Details of how this has been achieved are described in a separate contribution [7]. Although tuning of transition can not be done independent of injection and extraction parameters, issues specific to the operational aspects of crossing of the transition energy will be described here.

The machine complex was turned on with quadrupole pumping in the Booster. The high frequency cavity is still being used, but the resulting dilution is smaller because of the reduced phase mismatch of the beam with respect to the $\mathrm{RF}$ in the AGS. As in previous years the gap volts have been reduced during the ramping of the transition quadrupoles to minimize the momentum spread, which is estimated $\Delta p / p= \pm .6 \%$ at the time the transition jump occurs. Further the three sextupoles were powered with a current ramp similar to the ramp function in the transition quadrupoles. Because of the anticipated momentum spread the maximum current for this ramp was set at $35 \mathrm{~A}$.

In previous years dipole and quadrupole magnets have been moved to minimize the effect of the jump system on the orbit [2]. Before this run the sextupole magnet at J13 was moved because beam loss data from the previous run had shown excessive beam loss at this location. At another location, downstream of the D20 RF cavity, where a similar problem existed a backleg winding bump was installed. Despitc all these efforts the optimum tuned machine was one where the orbit excursions at the maximum of the ramp of the transition quads were intentionally not minimized and included deviations as large as $10 \mathrm{~mm}$. Although beam losses downstream of the D20 cavity have been reduced, they are still problematic.

When the machine was routinely running above $60 \mathrm{TP}$ it was observed that the transition losses were varying from cycle to cycle from $\sim 2$ to $\sim 4$ TP. A wall monitor mountain range program was modified such to provide integration of the individual bunch areas during the period around transition. This application revealed that most of the varying beam loss occurred on the last transfer from the Booster. Possible explanation for this behaviour is the fact that the last transfer has the highest intensity and the shortest exposure time to the high frequency cavity.

The performance of the transition jump system has improved during the recent run over previous years. On average beam losses have been reduced. while running at higher intensities. Because of the complexity of tuning the machine to the high intensities, it is not possible to identify, which of the new strategies has contributed most. but both. described in the above sections, will be pursued in coming runs.
The creation and evolution of the transition jump scheme has been driven by high intensity proton operation. The sextupole correction, in combination with the jump system, will also be explored further, when the AGS will be used as injector for RHIC, because of the desired low emittance for collider operation.

\section{REFERENCES}

[1] M.J. Syphers, et al., 'The AGS $\gamma_{t}$-jump system', BNL Report 60824

[2] W.K. van Asselt, et al., Proc.IEEE 1995 Particle Accelerator Conference, 95CH35843, 3022

[3] L.A. Ahrens, et al., Proc. 1994 EPAC, 485

[4] M. Blaskiewicz, et al.. Proc. IEEE 1995 Particle Accelerator Conference, 95CH35843, 383

[5] J. Wei, et al., Proc. IEEE 1995 Particle Accelerator Conference, 95CH35843, 3334

[6] C. Ankenbrandt, et al., Phys. Rev. ST, Accelerators and beams 1 (1998)030101

[7] L.A. Ahrens, et al., High intensity performance of the Brookhaven AGS, These Proceedings. 